\title{
Article \\ Existence of Solutions and Hyers-Ulam Stability for a Coupled System of Nonlinear Fractional Differential Equations with $p$-Laplacian Operator
}

\author{
Jing Shao ${ }^{1, *}$ and Boling Guo ${ }^{2}$ \\ 1 Department of Mathematics, Jining University, Qufu 273155, China \\ 2 Institute of Applied Physics and Computational Mathematics, Beijing 100088, China; gbl@iapcm.ac.cn \\ * Correspondence: shaojing99500@163.com
}

Citation: Shao, J.; Guo, B. Existence of Solutions and Hyers-Ulam Stability for a Coupled System of Nonlinear Fractional Differential Equations with $p$-Laplacian Operator. Symmetry 2021, 13, 1160. https:// doi.org/10.3390/sym13071160

Academic Editor: Juan Luis García Guirao and Sergei D. Odintsov

Received: 10 June 2021

Accepted: 25 June 2021

Published: 28 June 2021

Publisher's Note: MDPI stays neutral with regard to jurisdictional claims in published maps and institutional affiliations.

Copyright: (c) 2021 by the authors. Licensee MDPI, Basel, Switzerland. This article is an open access article distributed under the terms and conditions of the Creative Commons Attribution (CC BY) license (https:// creativecommons.org/licenses/by/ $4.0 /)$.

\begin{abstract}
In this paper, the existence and uniqueness of solutions to a coupled formally symmetric system of fractional differential equations with nonlinear $p$-Laplacian operator and nonlinear fractional differential-integral boundary conditions are obtained by using the matrix eigenvalue method. The Hyers-Ulam stability of the coupled formally symmetric system is also presented with certain growth conditions. By using the topological degree theory and nonlinear functional analysis methods, some sufficient conditions for the existence and uniqueness of solutions to this coupled formally symmetric system are established. Examples are provided to verify our results.
\end{abstract}

Keywords: existence and uniqueness of solutions; coupled system; Hyers-Ulam stability; topological degree theory; $p$-Laplacian

\section{Introduction}

Symmetry is an important form of many things in nature and society; many of the differential equations we studied are symmetric. Among these equations, the fractional differential equation is one of the important fields that has profound theories and wide applications in modern mathematics. Mathematical models of fractional differential equations are at the heart of quantitative descriptions of a large number of physical systems, including engineering, plasma physics, aerodynamics, electrical circuits and many other fields. The existence and stability of solutions for fractional differential equations are studied as one of the key techniques for solving physical systems (see [1-5] and references therein). The existence and uniqueness of solutions for fractional differential equations are investigated usually by using classical fixed point theory. Various kinds of stabilities have been established, such as Lyapunov stability, Mittag-Leffler stability and exponential stability, see ([6-9] for details). These stability results have attracted a lot of attention in recent years as they arise naturally in various areas of applications.

The stability of functional equations derived from the stability problem of group homomorphism was first proposed by Ulam in 1940. In 1941, Hyers solves the stability problem of additive mappings over Banach spaces. Since then, Hyers-Ulam stability has developed rapidly. These stability results are widely used in stochastic analysis, financial mathematics and actuarial science. As is known to all, it is difficult and time-consuming to calculate the Lyapunov stability for some nonlinear fractional differential equations, and it is also a challenge to construct the exact Lyapunov function. Hyers-Ulam stability is just suitable for nonlinear fractional differential equations dealing with this situation. A significant number of researchers have devoted to not only Hyers-Ulam stability but also the existence and uniqueness of solutions of fractional differential equations.

In 1983, Leibenson [10] introduced a differential equation with the $p$-Laplacian operator, which models the turbulent flow in a porous medium. The classical nonlinear $p$-Laplacian operator is defined as 


$$
\Phi_{p}(s)=|s|^{p-2} s, p>1, \frac{1}{p}+\frac{1}{q}=1, \Phi_{q}(s)=\Phi_{p}^{-1} .
$$

Henceforth, differential equations with a $p$-Laplacian operator are widely applied to different fields of physics and natural phenomena, for example, mechanics, dynamical systems, biophysics, plasmaphysics, material science, and electrodynamics (see [10-12] and the references therein).

The existence and Hyers-Ulam stability of solutions of fractional differential equations with $p$-Laplacian has attracted much attention in recent years. In 2014, using the LeggettWilliam fixed point theorem, Lu et al. [13] obtained the existence of two or three positive solutions of fractional differential equations with $p$-Laplacian operator. K. P. Prasad et al. [4] discuss the existence of positive solutions for the coupled system of the fractional order boundary value problem with $p$-Laplacian operator in 2016. In 2017, H. Khan et al. [14] investigate the existence, uniqueness and Hyers-Ulam stability for the following coupled system of fractional differential equations with $p$-Laplacian operator

$$
\left\{\begin{array}{l}
D_{0^{+}}^{\beta_{1}}\left(\Phi_{p}\left(D_{0^{+}}^{\alpha_{1}} v(t)\right)\right)=-\psi_{1}(t, z(t)), \\
D_{0^{+}}^{\beta_{2}}\left(\Phi_{p}\left(D_{0^{+}}^{\alpha_{2}} z(t)\right)\right)=-\psi_{2}(t, v(t)), \\
\left.D_{0^{+}}^{\alpha_{1}} v(t)\right|_{t=0}=0=\left.\left(\Phi_{p}\left(D_{0_{+}}^{\alpha_{1}} v(t)\right)\right)^{\prime}\right|_{t=0}=\left.D_{0^{+}}^{\delta_{1}} v(t)\right|_{t=\eta_{1}}, \\
v(1)=\left.\frac{\Gamma\left(2-\delta_{1}\right)}{\eta_{1}^{1-\delta_{1}}} \mathcal{J}^{\alpha_{1}-\delta_{1}} \Phi_{q}\left(\mathcal{J}_{0^{+}}^{\beta_{1}} \psi_{1}(t, z(t))\right)\right|_{t=\eta_{1}}, \\
\left.D_{0^{+}}^{\alpha_{2}} z(t)\right|_{t=0}=0=\left.\left(\Phi_{p}\left(D_{0_{+}}^{\alpha_{2}} z(t)\right)\right)^{\prime}\right|_{t=0}=\left.D_{0^{+}}^{\delta_{2}} z(t)\right|_{t=\eta_{2}}, \\
v(1)=\left.\frac{\Gamma\left(2-\delta_{2}\right)}{\eta_{2}^{1-\delta_{2}}} \mathcal{J}^{\alpha_{2}-\delta_{2}} \Phi_{q}\left(\mathcal{J}_{0^{+}}^{\beta_{2}} \psi_{2}(t, v(t))\right)\right|_{t=\eta_{2},}
\end{array}\right.
$$

where $t \in[0,1], \eta_{i}, \delta_{i} \in(0,1), \alpha_{i}, \beta_{i} \in(1,2]$, for $i=1,2$. Using topological degree theory and a Lerray-Schauder-type fixed point theorem, H. Khan et al. [15] studied the HyersUlam stability for this coupled system with the different initial boundary conditions for $\alpha_{i}, \beta_{i} \in(2,3)$. The nonlinear $p$-Laplacian operator is defined as that in (1).

A. Khan et al. [6] discuss the existence, uniqueness and Hyers-Ulam stability of solutions to a coupled system of fractional differential equations with nonlinear $p$-Laplacian operator

$$
\left\{\begin{array}{l}
D^{\beta_{1}}\left(\Phi_{p}\left(D^{\alpha_{1}} \mu(t)\right)\right)+\mathcal{F}_{1}(x, v(x))=0, x \in(0,1), \\
D^{\beta_{2}}\left(\Phi_{p}\left(D^{\alpha_{2}} v(t)\right)\right)+\mathcal{F}_{2}(x, \mu(x))=0, x \in(0,1), \\
\left.D^{\alpha_{1}} \mu(x)\right|_{x=0}=0=\left.\mu(x)\right|_{x=0}=\left.\mu^{\prime \prime}(x)\right|_{x=0}, \\
\left.\mu^{\prime}(x)\right|_{x=1}=\eta_{1} I_{P}^{\gamma_{1}} \psi_{1}(\mu), \\
\left.D^{\alpha_{2}} v(x)\right|_{x=0}=0=\left.v(x)\right|_{x=0}=\left.v^{\prime \prime}(x)\right|_{x=0}, \\
\left.v^{\prime}(x)\right|_{x=1}=\eta_{2} I_{P}^{\gamma_{2}} \psi_{2}(\mu),
\end{array}\right.
$$

where $\Phi_{p}$ is $p$-Laplacian operator and $2<\alpha_{i}<3,0<\beta_{i}<1, P, \eta_{i}, \gamma_{i}>0, \psi_{i} \in L[0,1]$, $i=1,2$ and $D^{\alpha_{i}}$ is the Caputo derivative of order $\alpha_{i}$.

Motivated by A. Khan [6] and H. Khan [14,15], this paper is devoted to study the existence, uniqueness and Hyers-Ulam stability of solutions to nonlinear coupled fractional differential equations with $p$-Laplacian operator of the form 


$$
\left\{\begin{array}{l}
D_{0}^{\beta_{1}}\left(L_{p}\left(D_{0}^{\alpha_{1}} x(t)\right)\right)-\mathcal{F}_{1}(t) x(t)=\Phi(t, x(t), y(t)), \\
D_{0}^{\beta_{2}}\left(L_{p}\left(D_{0}^{\alpha_{2}} y(t)\right)\right)-\mathcal{F}_{2}(t) y(t)=\Psi(t, x(t), y(t)), t \in \mathcal{T}, \\
\left.D_{0}^{\alpha_{1}} x(t)\right|_{t=0}=0,\left.x(t)\right|_{t=0}=0,\left.x^{\prime \prime}(t)\right|_{t=0}=0 \\
\left.x^{\prime}(t)\right|_{t=1}=\frac{\eta_{1}}{\Gamma\left(\gamma_{1}\right)} \int_{0}^{b}(b-\xi)^{\gamma_{1}-1} \psi_{1}(x(\xi)) d \xi \\
\left.D_{0}^{\alpha_{2}} y(t)\right|_{t=0}=0,\left.y(t)\right|_{t=0}=0,\left.y^{\prime \prime}(t)\right|_{t=0}=0 \\
\left.y^{\prime}(t)\right|_{t=1}=\frac{\eta_{2}}{\Gamma\left(\gamma_{2}\right)} \int_{0}^{b}(b-\xi)^{\gamma_{2}-1} \psi_{2}(y(\xi)) d \xi
\end{array}\right.
$$

where $2<\alpha_{i} \leq 3,0<\beta_{i} \leq 1, P, \eta_{i}, \gamma_{i}>0, \psi_{i} \in L[0,1]$, and $D_{0}^{\alpha_{i}}$ and $D_{0}^{\beta_{i}}$ are the Caputo derivatives of order $\alpha_{i}$ and $\beta_{i}, i=1,2$, respectively. $L_{p}(s)=|s|^{p-2} s$ is $p$-Laplacian operator, where $\frac{1}{p}+\frac{1}{q}=1, L_{q}$ denotes inverse of $p$-Laplacian. $\mathcal{F}_{i}: \mathcal{T} \rightarrow \mathbb{R}$ are closed bounded and linear operators for any $t \in \mathcal{T}=[0,1]$, and $\Phi, \Psi: \mathcal{T} \times \mathbb{R} \times \mathbb{R} \rightarrow \mathbb{R}$ are continuous functions, $i=1,2$. For this purpose, we use the coincidence degree method and nonlinear functional analysis theory to deal with the existence and uniqueness of solutions and the matrix eigenvalue method in order to investigate Hyers-Ulam stability.

The paper is organized as follows. In Section 2, we provide some auxiliary results which will be used in the next sections. In Section 3, using the coincidence degree theory and nonlinear functional analysis methods, the existence result of coupled system (2) is established, then the existence and uniqueness of solutions are discussed using Banach fixed point theorem. In Section 4, the Hyers-Ulam stability of the solutions is investigated by using the matrix eigenvalue method with some nonlinear boundary conditions. The Section 5 is devoted to providing some examples to illustrate the application of our main results.

\section{Auxiliary Results}

In this paper, we revisit the problem of Hyers-Ulam stability of Banach space using the coincidence degree theory and nonlinear functional analysis methods. Let $\mathcal{H}_{1}$ be the space of all continuous functions $x:[0,1] \rightarrow \mathbb{R}$, endowing the norm $\sup _{t \in[0,1]}\{|x(t)|: x \in C[0,1]\}$. Then $\mathcal{H}_{1}$ is a Banach space under this norm, and, hence, their product space, denoted by $\mathcal{H}=\mathcal{H}_{1} \times \mathcal{H}_{1}$, is also a Banach space with norm $\|(x, y)\|=\|x\|+\|y\|$. Here, we recall some special definitions, theorems and Hyers-Ulam stability results from the literature [1,3,5,16-18], which we will use throughout this paper.

Definition 1. Let $\sigma \in \mathbb{R}^{+}$. for a given function $x:[a, \infty) \rightarrow \mathbb{R}$, then its $\sigma$-order fractional integral in the sense of Riemann-Liouville is given by

$$
I_{a}^{\sigma} x(t)=\frac{1}{\Gamma(\sigma)} \int_{a}^{t}(t-\xi)^{\sigma-1} x(\xi) d \xi
$$

such that the integral on the right side is pointwise defined on $\mathbb{R}^{+}$.

Definition 2. Let $x$ be a given function on closed interval $[a, b]$, then its fractional order derivative in the sense of Caputo is stated as

$$
{ }^{C} D_{a}^{\sigma} x(t)=\int_{a}^{t} \frac{(t-\xi)^{n-\sigma-1}}{\Gamma(n-\sigma)}\left(\frac{d^{n}}{d \xi^{n}} x(\xi)\right) d \xi, \sigma \in(n-1, n],
$$

where $[\sigma]=n-1$. In particular if $x$ is defined on the interval $[a, b]$ and $\sigma \in(0,1)$, then

$$
{ }^{c} D_{a}^{\sigma} x(t)=\frac{1}{\Gamma(1-\sigma)} \int_{a}^{t} \frac{x^{\prime}(\xi)}{(t-\xi)^{\sigma}} d \xi \text {, where } x^{\prime}(s)=\frac{d x(s)}{d s} .
$$


Theorem 1 ([18]). Let $\sigma \in[n-1, n)$. For $x \in C[a, b]$, the unique solution of ${ }^{C} D_{a}^{\sigma} x(t)=0$ has the form $x(t)=\sum_{k=0}^{[\sigma]} a_{k} t^{k}$, where $a_{k} \in \mathbb{R}, k=1,2,3, \cdots,[\sigma]$.

Theorem 2 ([18]). Let $\sigma \in[n-1, n)$. For $x \in C^{(n)}[a, b], I_{a}^{\sigma}\left[{ }^{C} D_{a}^{\sigma} x(t)\right]=x(t)+\sum_{k=0}^{[\sigma]} a_{k} t^{k}$, for some $a_{k} \in \mathbb{R}, k=1,2,3, \cdots,[\sigma]$.

Definition 3. Let the class of the all-bounded set of $\mathcal{H}$ be denoted by $\mathbb{B}$. The mapping $\varsigma: \mathbb{B} \rightarrow(0, \infty)$ for Kuratowski measure of noncompactness is defined as

$$
\varsigma(\mu)=\inf \{d>0: \mu \text { has a finite cover for sets of diameter } \leq d\} \text {, where } \mu \in \mathbb{B} \text {. }
$$

Theorem 3. The following are the characteristics of the measure $S$ :

(1) For relative compact $A$, the Kuratowski measure $\varsigma(A)=0$;

(2) Semi-norm $\varsigma$, that is $\varsigma(k A)=|k| \varsigma(A), k \in \mathbb{R}$, and $\varsigma\left(A_{1}+A_{2}\right) \leq \varsigma\left(A_{1}\right)+\varsigma\left(A_{2}\right)$;

(3) $A_{1} \subset A_{2}$ yields $\varsigma\left(A_{1}\right) ; \varsigma\left(A_{1} \cup A_{2}\right)=\sup \left\{\varsigma\left(A_{1}\right), \varsigma\left(A_{2}\right)\right\}$;

(4) $\varsigma(\operatorname{conv} A)=\varsigma(A)$;

(5) $\varsigma(\bar{A})=\varsigma(A)$.

Definition 4. Assume that $v: \vartheta \rightarrow \omega$ is a bounded and continuous mapping such that $\vartheta \subset \omega$, if there exists $\zeta \geq 0$ such that $\varsigma(v(\mu)) \leq \zeta \varsigma(\mu)$ for all bounded $\mu \subset \vartheta$, then $v$ is a $\varsigma$-Lipschitz.

Furthermore, $v$ is called strict $\zeta$-contraction under the condition $\zeta<1$.

Definition 5. The function $v$ is $\varsigma$-condensing if $\varsigma(\nu(\mu))<\varsigma(\mu)$ for all bounded $\mu \subset \vartheta$ with $\varsigma(\mu)>0$. Therefore $\varsigma(v(\mu)) \geq \varsigma(\mu)$ yields $\varsigma(\mu)=0$.

Furthermore, we call $v: \vartheta \rightarrow \omega$ is Lipschitz for $\zeta>0$, such that

$$
\|v(\mu)-v(\bar{\mu})\| \leq \zeta\|\mu-\bar{\mu}\|, \text { for all } \mu, \bar{\mu} \in \vartheta .
$$

The condition $\zeta<1$ yields that $v$ is a strict contraction.

Theorem 4. The mapping $v: \vartheta \rightarrow \omega$ is $\varsigma$-Lipschitz with constant $\zeta=0$ if and only if $v$ is compact.

Theorem 5. The mapping $v: \vartheta \rightarrow \omega$ is $\varsigma$-Lipschitz for some constant $\zeta$ if and only if $v$ is Lipschitz with constant $\zeta$.

Theorem 6 ([19]). Let $v: \mathcal{H} \rightarrow \mathcal{H}$ be a $\varsigma$-contraction and $\mathcal{Z}=\{z \in \mathcal{H}$ : there exist $0 \leq \lambda \leq 1$ such that $z=\lambda v(z)\}$. Under the condition that $\mathcal{Z} \subset \mathcal{H}$ is bounded for $r>0$ and $\mathcal{Z} \subset \mu_{r}(0)$, with degree

$$
\operatorname{deg}\left(I-\lambda v, \mu_{r}(0), 0\right)=1, \text { for every } \lambda \in[0,1] .
$$

Then, $v$ has at least one fixed point.

Theorem 7 ([20]). Let $L_{p}$ be a $p$-Laplacian operator. We have

(i) If $1<p \leq 2, x_{1} \cdot x_{2}>0$, and $\left|x_{1}\right|,\left|x_{2}\right| \geq M_{1}>0$, then

$$
\left|L_{p}\left(x_{1}\right)-L_{p}\left(x_{2}\right)\right| \leq(p-1) M_{1}^{p-2}\left|x_{1}-x_{2}\right| ;
$$

(ii) If $p>2$, and $\left|x_{1}\right|,\left|x_{2}\right| \leq M_{2}$, then

$$
\left|L_{p}\left(x_{1}\right)-L_{p}\left(x_{2}\right)\right| \leq(p-1) M_{2}^{p-2}\left|x_{1}-x_{2}\right| .
$$

Definition 6 ([16]). Let $u_{1}, u_{2}: \mathcal{H} \rightarrow \mathcal{H}$ be two operators defined on $\mathcal{H}$. Then the operator system provided by

$$
\left\{\begin{array}{l}
x(t)=u_{1}(x, y)(t) \\
y(t)=u_{2}(x, y)(t)
\end{array}\right.
$$


is called Hyers-Ulam stable if we can find $C_{i}>0, i=1,2,3,4$ such that for each $\varepsilon_{i}>0, i=1,2$, and for each solution $\left(x^{*}, y^{*}\right) \in \mathcal{H}$ of the inequalities given by

$$
\left\{\begin{array}{l}
\left\|x^{*}-\Theta_{1}\left(x^{*}, y^{*}\right)\right\| \leq \varepsilon_{1}, \\
\left\|y^{*}-\Theta_{2}\left(x^{*}, y^{*}\right)\right\| \leq \varepsilon_{2},
\end{array}\right.
$$

there exists a solution $(\bar{x}, \bar{y}) \in \mathcal{H}$ of system (3), which satisfies

$$
\left\{\begin{array}{l}
\left\|x^{*}-\bar{x}\right\| \leq C_{1} \varepsilon_{1}+C_{2} \varepsilon_{2}, \\
\left\|y^{*}-\bar{y}\right\| \leq C_{3} \varepsilon_{1}+C_{4} \varepsilon_{2} .
\end{array}\right.
$$

Definition 7. If $\lambda_{i}(i=1,2, \cdots, n)$ are the (real or complex) eigenvalues of a matrix $Q \in \mathbb{C}^{n \times n}$, then the spectral radius $\rho(Q)$ is defined by

$$
\rho(Q)=\max \left\{\left|\lambda_{i}\right| i=1,2, \cdots, n\right\} .
$$

Furthermore, the matrix will converge to zero if $\rho(Q)<1$.

Theorem 8 ([16]). Let $u_{1}, u_{2}: \mathcal{H} \rightarrow \mathcal{H}$ be two operators such that

$$
\left\{\begin{array}{l}
\left\|u_{1}(x, y)-u_{1}\left(x^{*}, y^{*}\right)\right\| \leq C_{1}\left\|x-x^{*}\right\|+C_{2}\left\|y-y^{*}\right\|, \\
\left\|u_{2}(x, y)-u_{2}\left(x^{*}, y^{*}\right)\right\| \leq C_{3}\left\|x-x^{*}\right\|+C_{4}\left\|y-y^{*}\right\|
\end{array}\right.
$$

for all $(x, y),\left(x^{*}, y^{*}\right) \in \mathcal{H}$. If the matrix $Q=\left(\begin{array}{ll}C_{1} & C_{2} \\ C_{3} & C_{4}\end{array}\right)$ converges to zero, then the fixed points corresponding to system (3) are Hyers-Ulam stable.

\section{Existence Results}

To come to our main results, we need the following hypotheses:

Hypothesis 1. The operators $\mathcal{F}_{i}: \mathcal{T} \rightarrow \mathbb{R}$ are closed bounded and linear for any $t \in \mathcal{T}=[0,1]$ and $i=1,2$. Denote $m_{1}=\max _{t \in \mathcal{T}}\left\{\left|\mathcal{F}_{1}(t)\right|\right\}, m_{2}=\max _{t \in \mathcal{T}}\left\{\left|\mathcal{F}_{2}(t)\right|\right\}$.

Hypothesis 2. The functions $\Phi, \Psi: \mathcal{T} \times \mathbb{R} \times \mathbb{R} \rightarrow \mathbb{R}$ are continuous. For all $(x, y),(\bar{x}, \bar{y}) \in \mathbb{R}$, $t \in \mathcal{T}$, there exist $m_{\Phi}, m_{\Psi}>0$, such that

$$
\begin{aligned}
& |\Phi(t, x(t), y(t))-\Phi(t, \bar{x}(t), \bar{y}(t))| \leq\left|m_{\Phi}(|x(t)-\bar{x}(t)|+|y(t)-\bar{y}(t)|)\right| \leq m_{\Phi}\|(x-\bar{x}, y-\bar{y})\|, \\
& |\Psi(t, x(t), y(t))-\Psi(t, \bar{x}(t), \bar{y}(t))| \leq\left|m_{\Psi}(|x(t)-\bar{x}(t)|+|y(t)-\bar{y}(t)|)\right| \leq m_{\Psi}\|(x-\bar{x}, y-\bar{y})\| .
\end{aligned}
$$

Hypothesis 3. The functions $\Phi$ and $\Psi$ satisfy the following growth conditions under the constants $\mathcal{C}_{\Phi}, \mathcal{C}_{\Psi}, M_{\Phi}, M_{\Psi}, q_{1} \in(0,1]$

$$
\begin{aligned}
& \|\Phi(t, x, y)\| \leq \mathcal{C}_{\Phi}|x|^{q_{1}}+M_{\Phi}, \\
& \|\Psi(t, x, y)\| \leq \mathcal{C}_{\Psi}|y|^{q_{1}}+M_{\Psi} .
\end{aligned}
$$

Hypothesis 4 . The nonlocal functions $\psi_{1}, \psi_{2}: \mathbb{R} \rightarrow \mathbb{R}$ satisfy the hypotheses that for any $(x, y)$, $(\bar{x}, \bar{y}) \in \mathbb{R}, t \in \mathcal{T}$, there exist $\mathbf{K}_{1}>0, \mathbf{K}_{2}>0$, such that

$$
\begin{aligned}
& \left|\psi_{1}(x)-\psi_{1}(\bar{x})\right| \leq \mathbf{K}_{1}\|x-\bar{x}\|, \\
& \left|\psi_{2}(y)-\psi_{2}(\bar{y})\right| \leq \mathbf{K}_{2}\|y-\bar{y}\| .
\end{aligned}
$$


Hypothesis 5. The nonlocal functions $\psi_{1}, \psi_{2}: \mathbb{R} \rightarrow \mathbb{R}$ satisfy the following growth conditions by the constants $\mathcal{C}_{v_{1}}, \mathcal{C}_{v_{2}}, M_{v_{1}}, M_{v_{2}}>0$ and $p_{1} \in[0,1)$ for $x, y \in \mathbb{R}$,

$$
\begin{aligned}
& \left|\psi_{1}(x)\right| \leq \mathcal{C}_{v_{1}}|x|^{p_{1}}+M_{v_{1}}, \\
& \left|\psi_{2}(y)\right| \leq \mathcal{C}_{v_{2}}|y|^{p_{1}}+M_{v_{2}} .
\end{aligned}
$$

Theorem 9. Assume that $z: \mathcal{T} \rightarrow \mathbb{R}$ and $\mathcal{F}_{1}: \mathcal{T} \rightarrow \mathbb{R}$ be bounded linear operators, then the solution of

$$
\begin{aligned}
& D_{0}^{\beta_{1}} L_{b}\left(D_{0}^{\alpha_{1}} x(t)\right)-\mathcal{F}_{1}(t) x(t)=z(t), \beta_{1} \in(0,1], \alpha_{1} \in(2,3], t \in \mathcal{T}, \\
& \left.x(t)\right|_{t=0}=0,\left.x^{\prime \prime}(t)\right|_{t=0}=0,\left.D_{0}^{\alpha_{1}} x(t)\right|_{t=0}=0, \\
& \left.x^{\prime}(t)\right|_{t=1}=\eta_{1} I_{b}^{\gamma_{1}} \psi_{1}(x)=\frac{\eta_{1}}{\Gamma\left(\gamma_{1}\right)} \int_{0}^{b}(b-\xi)^{\gamma_{1}-1} \psi_{1}(x(\xi)) d \xi
\end{aligned}
$$

is given by

$$
\begin{aligned}
x(t)= & \int_{0}^{1} G_{\alpha_{1}}(t, \xi) \cdot L_{q}\left(I_{0}^{\beta_{1}}\left(\mathcal{F}_{1}(\xi) x(\xi)+z(\xi)\right)\right) d \xi \\
& +\frac{\eta_{1} t}{\Gamma\left(\gamma_{1}\right)} \int_{0}^{b}(b-\xi)^{\gamma_{1}-1} \psi_{1}(x(\xi)) d \xi,
\end{aligned}
$$

where $G_{\alpha_{1}}(t, \xi)$ is Green's function, given by

$$
G_{\alpha_{1}}(t, \xi)=\left\{\begin{array}{l}
\frac{(t-\xi)^{\alpha_{1}-1}}{\Gamma\left(\alpha_{1}\right)}-\frac{t(1-\xi)^{\alpha_{1}-2}}{\Gamma\left(\alpha_{1}-1\right)}, 0 \leq \xi \leq t \leq 1 \\
-\frac{t(1-\xi)^{\alpha_{1}-2}}{\Gamma\left(\alpha_{1}-1\right)}, 0 \leq t \leq \xi \leq 1
\end{array}\right.
$$

Proof. Since

$$
D_{0}^{\beta_{1}} L_{b}\left(D_{0}^{\alpha_{1}} x(t)\right)-\mathcal{F}_{1}(t) x(t)=z(t), t \in[0,1], \beta_{1} \in(0,1], \alpha_{1} \in(2,3] .
$$

Applying the operator $I_{0}^{\beta_{1}}$ on (5) and using Theorem 2, we can obtain the following integral form as

$$
L_{b}\left(D_{0}^{\alpha_{1}} x(t)\right)=I_{0}^{\beta_{1}}\left(\mathcal{F}_{1}(t) x(t)+z(t)\right)+a_{0} .
$$

Using the initial condition $\left.D_{0}^{\alpha_{1}} x(t)\right|_{t=0}=0$, we have $a_{0}=0$.

Furthermore,

$$
D_{0}^{\alpha_{1}} x(t)=L_{q}\left[I_{0}^{\beta_{1}}\left(\mathcal{F}_{1}(t) x(t)+z(t)\right)\right] .
$$

Using Theorem 2 and applying the operator $I_{0}^{\alpha_{1}}$ on (6), we have

$$
x(t)=I_{0}^{\alpha_{1}}\left\{L_{q}\left[I_{0}^{\beta_{1}}\left(\mathcal{F}_{1}(t) x(t)+z(t)\right)\right]\right\}+a_{1}+a_{2} t+a_{3} t^{2} .
$$

By using the conditions $\left.x(t)\right|_{t=0}=0$ and $\left.x^{\prime \prime}(t)\right|_{t=0}=0$ in (7), we obtain $a_{1}=a_{3}=0$. We also obtain

$$
x^{\prime}(t)=\frac{1}{\Gamma\left(\alpha_{1}-1\right)} \int_{0}^{t}(t-\xi)^{\alpha_{1}-2} L_{q}\left[I_{0}^{\beta_{1}}\left(\mathcal{F}_{1}(\xi) x(\xi)+z(\xi)\right)\right] d \xi+a_{2} .
$$

If $\left.x^{\prime}(t)\right|_{t=1}=\eta_{1} I_{b}^{\gamma_{1}} \psi_{1}(x)$ in (8), then

$a_{2}=\frac{\eta_{1}}{\Gamma\left(\gamma_{1}\right)} \int_{0}^{b}(b-\xi)^{\gamma_{1}-1} \psi_{1}(x(\xi)) d \xi-\frac{1}{\Gamma\left(\alpha_{1}-1\right)} \int_{0}^{1}(1-\xi)^{\alpha_{1}-2} \cdot L_{q}\left(I_{0}^{\beta_{1}}\left(\mathcal{F}_{1}(\xi) x(\xi)+z(\xi)\right)\right) d \xi$.

By substituting the values of $a_{1}, a_{2}, a_{3}$ in (7), we obtain the following integral equation: 


$$
\begin{aligned}
x(t)= & \frac{1}{\Gamma\left(\alpha_{1}\right)} \int_{0}^{t}(t-\xi)^{\alpha_{1}-1} \cdot L_{q}\left(I_{0}^{\beta_{1}}\left(\mathcal{F}_{1}(\xi) x(\xi)+z(\xi)\right)\right) d \xi \\
& -\frac{t}{\Gamma\left(\alpha_{1}-1\right)} \int_{0}^{1}(1-\xi)^{\alpha_{1}-2} \cdot L_{q}\left(I_{0}^{\beta_{1}}\left(\mathcal{F}_{1}(\xi) x(\xi)+z(\xi)\right)\right) d \xi+\frac{\eta_{1} t}{\Gamma\left(\gamma_{1}\right)} \int_{0}^{b}(b-\xi)^{\gamma_{1}-1} \psi_{1}(x(\xi)) d \xi \\
= & \int_{0}^{1} G_{\alpha_{1}}(t, \xi) \cdot L_{q}\left(I_{0}^{\beta_{1}}\left(\mathcal{F}_{1}(\xi) x(\xi)+z(\xi)\right)\right) d \xi+\frac{\eta_{1} t}{\Gamma\left(\gamma_{1}\right)} \int_{0}^{b}(b-\xi)^{\gamma_{1}-1} \psi_{1}(x(\xi)) d \xi,
\end{aligned}
$$

where $G_{\alpha_{1}}(t, \xi)$ is defined as (4). This completes the proof.

According to Theorem 9, the equivalent system of Hammerstein-type integral equations corresponding to coupled system (2) is given by

$$
\begin{aligned}
& x(t)=\int_{0}^{1} G_{\alpha_{1}}(t, \xi) \cdot L_{q}\left(I_{0}^{\beta_{1}}\left(\mathcal{F}_{1}(\xi) x(\xi)+\Phi(\xi, x(\xi), y(\xi))\right)\right) d \xi+\frac{\eta_{1} t}{\Gamma\left(\gamma_{1}\right)} \int_{0}^{b}(b-\xi)^{\gamma_{1}-1} \psi_{1}(x(\xi)) d \xi, \\
& y(t)=\int_{0}^{1} G_{\alpha_{2}}(t, \xi) \cdot L_{q}\left(I_{0}^{\beta_{2}}\left(\mathcal{F}_{2}(\xi) x(\xi)+\Psi(\xi, x(\xi), y(\xi))\right)\right) d \xi+\frac{\eta_{2} t}{\Gamma\left(\gamma_{2}\right)} \int_{0}^{b}(b-\xi)^{\gamma_{2}-1} \psi_{2}(x(\xi)) d \xi,
\end{aligned}
$$

where $G_{\alpha_{1}}(t, \xi)$ is defined as $(4)$, and $G_{\alpha_{2}}(t, \xi)$ is defined by

$$
G_{\alpha_{2}}(t, \xi)=\left\{\begin{array}{l}
\frac{(t-\xi)^{\alpha_{2}-1}}{\Gamma\left(\alpha_{2}\right)}-\frac{t(1-\xi)^{\alpha_{2}-2}}{\Gamma\left(\alpha_{2}-1\right)}, 0 \leq \xi \leq t \leq 1, \\
-\frac{t(1-\xi)^{\alpha_{2}-2}}{\Gamma\left(\alpha_{2}-1\right)}, 0 \leq t \leq \xi \leq 1 .
\end{array}\right.
$$

Now, we consider a closed ball $\mathcal{B}_{r}=\{(x, y) \in \mathcal{H}:\|(x, y)\| \leq r\}$ and define operators $U=\left(U_{1}, U_{2}\right), V=\left(V_{1}, V_{2}\right)$ on $\mathcal{B}_{r}$ as

$$
\left\{\begin{array}{l}
U_{1}(x, y)(t)=\int_{0}^{1} G_{\alpha_{1}}(t, \xi) \cdot L_{q}\left(I_{0}^{\beta_{1}}\left(\mathcal{F}_{1}(\xi) x(\xi)+\Phi(\xi, x(\xi), y(\xi))\right)\right) d \xi \\
U_{2}(x, y)(t)=\int_{0}^{1} G_{\alpha_{2}}(t, \xi) \cdot L_{q}\left(I_{0}^{\beta_{2}}\left(\mathcal{F}_{2}(\xi) y(\xi)+\Psi(\xi, x(\xi), y(\xi))\right)\right) d \xi
\end{array}\right.
$$

and

$$
\left\{\begin{array}{l}
V_{1} x(t)=\frac{\eta_{1} t}{\Gamma\left(\gamma_{1}\right)} \int_{0}^{b}(b-\xi)^{\gamma_{1}-1} \psi_{1}(x(\xi)) d \xi, \\
V_{2} y(t)=\frac{\eta_{2} t}{\Gamma\left(\gamma_{2}\right)} \int_{0}^{b}(b-\xi)^{\gamma_{2}-1} \psi_{2}(x(\xi)) d \xi .
\end{array}\right.
$$

Then, the operator equation of the Hammerstein-type integral system (9) is given by

$$
(x, y)=W(x, y)=U(x, y)+V(x, y) .
$$

So, the solution of system (9) is the fixed points of operator Equation (13).

Theorem 10. Under the hypotheses $\left(H_{4}\right)$ and $\left(H_{5}\right)$ and if $\mathcal{C}_{v}<1$, the operator $V$ is $\varsigma$-Lipschitz and satisfies the growth condition given by

$$
\|V(x, y)\| \leq \mathcal{C}_{v}^{*}\|(x, y)\|^{p_{1}}+M_{v}, \forall(x, y) \in \mathcal{H},
$$

where

$$
\begin{aligned}
& \mathcal{C}_{v}=\max \left\{\frac{\eta_{1} b^{\gamma_{1}} \mathbf{K}_{1}}{\Gamma\left(\gamma_{1}+1\right)}, \frac{\eta_{2} b^{\gamma_{2}} \mathbf{K}_{2}}{\Gamma\left(\gamma_{2}+1\right)}\right\}, \\
& \mathcal{C}_{v}^{*}=\max \left\{\frac{\eta_{1} b^{\gamma_{1}} \mathcal{C}_{v_{1}}}{\Gamma\left(\gamma_{1}+1\right)}, \frac{\eta_{2} b^{\gamma_{2}} \mathcal{C}_{v_{2}}}{\Gamma\left(\gamma_{2}+1\right)}\right\},
\end{aligned}
$$


and

$$
M_{v}=\frac{\eta_{1} b^{\gamma_{1}} M_{v_{1}}}{\Gamma\left(\gamma_{1}+1\right)}+\frac{\eta_{2} b^{\gamma_{2}} M_{v_{2}}}{\Gamma\left(\gamma_{2}+1\right)} .
$$

Proof. Using the condition $\left(H_{4}\right)$ and $t \leq 1$, we have

$$
\begin{aligned}
\left|V_{1}(x)(t)-V_{1}(\bar{x})(t)\right| & =\left|\frac{\eta_{1} t}{\Gamma\left(\gamma_{1}\right)} \int_{0}^{b}(b-\xi)^{\gamma_{1}-1}\left[\psi_{1}(x(\xi))-\psi_{1}(\bar{x}(\xi))\right] d \xi\right| \\
& \leq \mathbf{K}_{1}\|x-\bar{x}\|\left|\frac{\eta_{1} t}{\Gamma\left(\gamma_{1}\right)} \int_{0}^{b}(b-\xi)^{\gamma_{1}-1} d \xi\right| \\
& =\frac{\eta_{1} b^{\gamma_{1}} \mathbf{K}_{1}}{\Gamma\left(\gamma_{1}+1\right)}\|x-\bar{x}\| .
\end{aligned}
$$

Similarly, we obtain

$$
\left|V_{2}(y)(t)-V_{2}(\bar{y})(t)\right| \leq \frac{\eta_{2} b^{\gamma_{2}} \mathbf{K}_{2}}{\Gamma\left(\gamma_{2}+1\right)}\|y-\bar{y}\| .
$$

Then,

$$
\begin{aligned}
\|V(x, y)-V(\bar{x}, \bar{y})\| & =\left\|V_{1}(x)-V_{2}(\bar{x})\right\|+\left\|V_{1}(y)-V_{2}(\bar{y})\right\| \\
& \leq \frac{\eta_{1} b^{\gamma_{1}} \mathbf{K}_{1}}{\Gamma\left(\gamma_{1}+1\right)}\|x-\bar{x}\|+\frac{\eta_{2} b^{\gamma_{2}} \mathbf{K}_{2}}{\Gamma\left(\gamma_{2}+1\right)}\|x-\bar{x}\| \\
& \leq \mathcal{C}_{v}\|(x-\bar{x}, y-\bar{y})\|,
\end{aligned}
$$

where $\mathcal{C}_{v}$ is defined by (15), $\mathcal{C}_{v} \in[0,1)$. Therefore, using Theorem 5 , the operator $V$ is $\zeta$-Lipschitz.

Next, to obtain the growth condition, using the condition $\left(H_{5}\right)$, we have

$$
\begin{aligned}
\left|V_{1}(x)(t)\right| & =\left|\frac{\eta_{1} t}{\Gamma\left(\gamma_{1}\right)} \int_{0}^{b}(b-\xi)^{\gamma_{1}-1} \psi_{1}(x(\xi)) d \xi\right| \\
& \leq \frac{\eta_{1}}{\Gamma\left(\gamma_{1}\right)} \int_{0}^{b}(b-\xi)^{\gamma_{1}-1}\left|\psi_{1}(x(\xi))\right| d \xi \\
& \leq \frac{\eta_{1}}{\Gamma\left(\gamma_{1}\right)} \int_{0}^{b}(b-\xi)^{\gamma_{1}-1}\left[\mathcal{C}_{v_{1}}|x|^{p_{1}}+M_{v_{1}}\right] d \xi .
\end{aligned}
$$

Then,

$$
\left\|V_{1}(x)\right\| \leq \frac{\eta_{1} b^{\gamma_{1}}}{\Gamma\left(\gamma_{1}+1\right)}\left[\mathcal{C}_{v_{1}}\|x\|^{p_{1}}+M_{v_{1}}\right] .
$$

Similarly, we have

$$
\left\|V_{2}(y)\right\| \leq \frac{\eta_{2} b^{\gamma_{2}}}{\Gamma\left(\gamma_{2}+1\right)}\left[\mathcal{C}_{v_{2}}\|y\|^{p_{1}}+M_{v_{2}}\right] .
$$

Hence, (18) and (19) imply that

$$
\begin{aligned}
\|V(x, y)\| & =\left\|V_{1}(x)\right\|+\left\|V_{2}(y)\right\| \\
& \leq \frac{\eta_{1} b^{\gamma_{1}}}{\Gamma\left(\gamma_{1}+1\right)}\left[\mathcal{C}_{v_{1}}\|x\|^{p_{1}}+M_{v_{1}}\right]+\frac{\eta_{2} b^{\gamma_{2}}}{\Gamma\left(\gamma_{2}+1\right)}\left[\mathcal{C}_{v_{2}}\|y\|^{p_{1}}+M_{v_{2}}\right] \\
& =\frac{\eta_{1} b^{\gamma_{1}} \mathcal{C}_{v_{1}}}{\Gamma\left(\gamma_{1}+1\right)}\|x\|^{p_{1}}+\frac{\eta_{2} b^{\gamma_{2}} \mathcal{C}_{v_{2}}}{\Gamma\left(\gamma_{2}+1\right)}\|y\|^{p_{1}}+\frac{\eta_{1} b^{\gamma_{1}} M_{v_{1}}}{\Gamma\left(\gamma_{1}+1\right)}+\frac{\eta_{2} b^{\gamma_{2}} M_{v_{2}}}{\Gamma\left(\gamma_{2}+1\right)} \\
& \leq \mathcal{C}_{v}^{*}\|(x, y)\|^{p_{1}}+M_{v},
\end{aligned}
$$

where $\mathcal{C}_{v}^{*}, M_{v}$ are defined as (16) and (17). This completes the proof. 
Theorem 11. Under the hypotheses $\left(H_{1}\right)$ and $\left(H_{3}\right)$, the operator $U$ is continuous and satisfies the growth condition given by

$$
\|U(x, y)\| \leq(q-1) M_{1}^{q-2}\left(A_{1}\|(x, y)\|^{q_{1}}+A_{2}\|(x, y)\|+A_{3}\right),
$$

where

$$
\begin{aligned}
& A_{1}=\max _{(x, y) \in \mathcal{B}_{r}}\left\{\frac{\mathcal{C}_{\Phi}}{\Gamma\left(\alpha_{1}+\beta_{1}\right)}, \frac{\mathcal{C}_{\Psi}}{\Gamma\left(\alpha_{2}+\beta_{2}\right)}\right\}, \\
& A_{2}=\max _{(x, y) \in \mathcal{B}_{r}}\left\{\frac{m_{1}}{\Gamma\left(\alpha_{1}+\beta_{1}\right)}, \frac{m_{2}}{\Gamma\left(\alpha_{2}+\beta_{2}\right)}\right\},
\end{aligned}
$$

and

$$
A_{3}=\max _{(x, y) \in \mathcal{B}_{r}}\left\{\frac{M_{\Phi}}{\Gamma\left(\alpha_{1}+\beta_{1}\right)}, \frac{M_{\Psi}}{\Gamma\left(\alpha_{2}+\beta_{2}\right)}\right\} .
$$

Proof. To prove the continuity of operator $U$, we construct a sequence $\left\{\left(x_{n}, y_{n}\right)\right\}$ in $\mathcal{B}_{r}$ with $\left(x_{n}, y_{n}\right) \rightarrow(x, y)$ as $n \rightarrow \infty$. Then, using Theorem 7 and the condition $\left(H_{1}\right)$, we have

$$
\begin{aligned}
\left|U_{1}\left(x_{n}, y_{n}\right)(t)-U_{1}(x, y)(t)\right|= & \mid \int_{0}^{1} G_{\alpha_{1}}(t, \xi) L_{q}\left(I_{0}^{\beta_{1}}\left(\mathcal{F}_{1}(\xi) x_{n}(\xi)+\Phi\left(\xi, x_{n}(\xi), y_{n}(\xi)\right)\right)\right) d \xi \\
& -\int_{0}^{1} G_{\alpha_{1}}(t, \xi) L_{q}\left(I_{0}^{\beta_{1}}\left(\mathcal{F}_{1}(\xi) x(\xi)+\Phi(\xi, x(\xi), y(\xi))\right)\right) d \xi \mid \\
\leq & (q-1) M_{1}^{q-2} \int_{0}^{1} G_{\alpha_{1}}(t, \xi)\left(\left|I_{0}^{\beta_{1}} \mathcal{F}_{1}(\xi) x_{n}(\xi)-I_{0}^{\beta_{1}} \mathcal{F}_{1}(\xi) x(\xi)\right|\right. \\
& \left.+\left|I_{0}^{\beta_{1}} \Phi\left(\xi, x_{n}(\xi), y_{n}(\xi)\right)-I_{0}^{\beta_{1}} \Phi(\xi, x(\xi), y(\xi))\right|\right) d \xi \\
\leq & (q-1) M_{1}^{q-2} m_{1} \int_{0}^{1} G_{\alpha_{1}}(t, \xi)\left|I_{0}^{\beta_{1}} x_{n}(\xi)-I_{0}^{\beta_{1}} x(\xi)\right| d \xi \\
& +(q-1) M_{1}^{q-2} \int_{0}^{1} G_{\alpha_{1}}(t, \xi)\left|I_{0}^{\beta_{1}} \Phi\left(\xi, x_{n}(\xi), y_{n}(\xi)\right)-I_{0}^{\beta_{1}} \Phi(\xi, x(\xi), y(\xi))\right| d \xi .
\end{aligned}
$$

Due to the continuity of $\Phi$, one has $\Phi\left(\xi, x_{n}(\xi), y_{n}(\xi)\right) \rightarrow \Phi(\xi, x(\xi), y(\xi))$ as $n \rightarrow \infty$. Using the Lebesgue-dominated convergent theorem, we have $\mid I_{0}^{\beta_{1}} \Phi\left(\xi, x_{n}(\xi), y_{n}(\xi)\right)-$ $I_{0}^{\beta_{1}} \Phi(\xi, x(\xi), y(\xi)) \mid \rightarrow 0$ as $n \rightarrow \infty$. We also obtain $I_{0}^{\beta_{1}} x_{n}(\xi) \rightarrow I_{0}^{\beta_{1}} x(\xi)$ as $n \rightarrow \infty$. So, $\left|U_{1}\left(x_{n}, y_{n}\right)(t)-U_{1}(x, y)(t)\right| \rightarrow 0$ as $n \rightarrow \infty$. Hence, $U_{1}$ is continuous. Similarly, we obtain

$$
\begin{aligned}
\left|U_{2}\left(x_{n}, y_{n}\right)(t)-U_{2}(x, y)(t)\right| \leq & (q-1) M_{1}^{q-2} m_{2} \int_{0}^{1} G_{\alpha_{2}}(t, \xi)\left|I_{0}^{\beta_{2}} y_{n}(\xi)-I_{0}^{\beta_{2}} y(\xi)\right| d \xi \\
& +(q-1) M_{1}^{q-2} \int_{0}^{1} G_{\alpha_{2}}(t, \xi)\left|I_{0}^{\beta_{2}} \Psi\left(\xi, x_{n}(\xi), y_{n}(\xi)\right)-I_{0}^{\beta_{2}} \Psi(\xi, x(\xi), y(\xi))\right| d \xi .
\end{aligned}
$$

Using the continuity of $\Psi$ and $I_{0}^{\beta_{2}} y_{n}(\xi) \rightarrow I_{0}^{\beta_{2}} y(\xi)$ as $n \rightarrow \infty$, we obtain $\mid U_{2}\left(x_{n}, y_{n}\right)(t)-$ $U_{2}(x, y)(t) \mid \rightarrow 0$ as $n \rightarrow \infty$ similarly. Hence, $U_{2}$ is continuous. Due to $\mid U\left(x_{n}, y_{n}\right)(t)-$ $U(x, y)(t)|=| U_{1}\left(x_{n}, y_{n}\right)(t)-U_{1}(x, y)(t)|+| U_{2}\left(x_{n}, y_{n}\right)(t)-U_{2}(x, y)(t) \mid$, we have that the operator $U$ is continuous.

Next, for growth condition (20), using $\left(H_{1}\right),\left(H_{3}\right)$ and Theorem 7 , we have

$$
\begin{aligned}
\left|U_{1}(x, y)(t)\right| & \leq(q-1) M_{1}^{q-2}\left|\int_{0}^{1} G_{\alpha_{1}}(t, \xi) I_{0}^{\beta_{1}} \mathcal{F}_{1}(\xi) x(\xi) d \xi\right|+(q-1) M_{1}^{q-2}\left|\int_{0}^{1} G_{\alpha_{1}}(t, \xi) I_{0}^{\beta_{1}} \Phi(\xi, x(\xi), y(\xi)) d \xi\right| \\
& \leq(q-1) M_{1}^{q-2} m_{1}\left|\frac{t^{\alpha_{1}+\beta_{1}}-t\left(\alpha_{1}-1\right)}{\Gamma\left(\alpha_{1}+\beta_{1}\right)}\right|\|x\|+(q-1) M_{1}^{q-2}\left|\frac{t^{\alpha_{1}+\beta_{1}-t\left(\alpha_{1}-1\right)}}{\Gamma\left(\alpha_{1}+\beta_{1}\right)}\right|\left(\mathcal{C}_{\Phi}\|x\|^{q_{1}}+M_{\Phi}\right) \\
& \leq \frac{(q-1) M_{1}^{q-2} m_{1}}{\Gamma\left(\alpha_{1}+\beta_{1}\right)}\|x\|+\frac{(q-1) M_{1}^{q-2}}{\Gamma\left(\alpha_{1}+\beta_{1}\right)}\left(\mathcal{C}_{\Phi}\|x\|^{q_{1}}+M_{\Phi}\right)
\end{aligned}
$$


which implies that

$$
\left\|U_{1}(x, y)\right\| \leq \frac{(q-1) M_{1}^{q-2}}{\Gamma\left(\alpha_{1}+\beta_{1}\right)}\left(\mathcal{C}_{\Phi}\|x\|^{q_{1}}+m_{1}\|x\|+M_{\Phi}\right),
$$

and similarly

$$
\left\|U_{2}(x, y)\right\| \leq \frac{(q-1) M_{1}^{q-2}}{\Gamma\left(\alpha_{2}+\beta_{2}\right)}\left(\mathcal{C}_{\Psi}\|y\|^{q_{1}}+m_{2}\|y\|+M_{\Psi}\right) .
$$

It follows from (24) and (25) that

$$
\begin{aligned}
\|U(x, y)\|= & \left\|U_{1}(x, y)\right\|+\left\|U_{2}(x, y)\right\| \\
\leq & (q-1) M_{1}^{q-2}\left(\frac{\mathcal{C}_{\Phi}}{\Gamma\left(\alpha_{1}+\beta_{1}\right)}\|x\|^{q_{1}}+\frac{\mathcal{C}_{\Psi}}{\Gamma\left(\alpha_{2}+\beta_{2}\right)}\|y\|^{q_{1}}\right) \\
& +(q-1) M_{1}^{q-2}\left(\frac{m_{1}}{\Gamma\left(\alpha_{1}+\beta_{1}\right)}\|x\|+\frac{m_{2}}{\Gamma\left(\alpha_{2}+\beta_{2}\right)}\|y\|\right) \\
& +(q-1) M_{1}^{q-2}\left(\frac{M_{\Phi}}{\Gamma\left(\alpha_{1}+\beta_{1}\right)}+\frac{M_{\Psi}}{\Gamma\left(\alpha_{2}+\beta_{2}\right)}\right) \\
\leq & (q-1) M_{1}^{q-2}\left(A_{1}\|(x, y)\|^{q_{1}}+A_{2}\|(x, y)\|+A_{3}\right),
\end{aligned}
$$

where $A_{1}, A_{2}, A_{3}$ are defined as (21)-(23). This completes the proof.

Theorem 12. Under the hypotheses $\left(H_{1}\right)$ and $\left(H_{3}\right)$, the operator $U: \mathcal{H} \rightarrow \mathcal{H}$ is compact and $\varsigma$-Lipschitz with constant zero.

Proof. With the assumption $\left(H_{1}\right)$ and $\left(H_{3}\right)$, Theorem 11 implies that the operator $U$ is bounded. Let $\mathcal{D}$ be a bounded subset of $\mathcal{B}_{r}$. For the given sequence $\left\{\left(x_{n}, y_{n}\right)\right\} \subset \mathcal{D}$ and for any $t_{1}, t_{2} \in[0,1]$, we obtain

$$
\begin{aligned}
& \left|U_{1}\left(x_{n}, y_{n}\right)\left(t_{1}\right)-U_{1}\left(x_{n}, y_{n}\right)\left(t_{2}\right)\right| \\
& \leq(q-1) M_{1}^{q-2}\left|\int_{0}^{1}\left(G_{\alpha_{1}}\left(t_{1}, \xi\right)-G_{\alpha_{1}}\left(t_{2}, \xi\right)\right) \frac{1}{\Gamma\left(\beta_{1}\right)} \int_{0}^{\xi}(\xi-\tau)^{\beta_{1}-1} d \tau d \xi\right|\left(\mathcal{C}_{\Phi}\left\|x_{n}\right\|^{q_{1}}+m_{1}\left\|x_{n}\right\|+M_{\Phi}\right) .
\end{aligned}
$$

By simplification, we obtain

$$
\begin{aligned}
& \left|U_{1}\left(x_{n}, y_{n}\right)\left(t_{1}\right)-U_{1}\left(x_{n}, y_{n}\right)\left(t_{2}\right)\right| \\
\leq & (q-1) M_{1}^{q-2}\left[\frac{1}{\Gamma\left(\beta_{1}+1\right) \Gamma\left(\alpha_{1}\right)}\left|\int_{0}^{t_{1}}\left(t_{1}-\xi\right)^{\alpha_{1}-1} \xi^{\beta_{1}} d \xi-\int_{0}^{t_{2}}\left(t_{2}-\xi\right)^{\alpha_{1}-1} \xi^{\beta_{1}} d \xi\right|\right. \\
& \left.+\frac{1}{\Gamma\left(\beta_{1}+1\right) \Gamma\left(\alpha_{1}-1\right)}\left|\left(t_{2}-t_{1}\right) \int_{0}^{1}(1-\xi)^{\alpha_{1}-2} \xi^{\beta_{1}} d \xi\right|\right]\left(\left.\mathcal{C}_{\Phi}\left\|x_{n}\right\|\right|^{q_{1}}+m_{1}\left\|x_{n}\right\|+M_{\Phi}\right) \\
= & {\left[\frac{(q-1) M_{1}^{q-2} B\left(\alpha_{1}, \beta_{1}+1\right)}{\Gamma\left(\beta_{1}+1\right) \Gamma\left(\alpha_{1}\right)}\left|t_{1}^{\alpha_{1}+\beta_{1}}-t_{2}^{\alpha_{1}+\beta_{1}}\right|+\frac{(q-1) M_{1}^{q-2} B\left(\alpha_{1}-1, \beta_{1}+1\right)}{\Gamma\left(\beta_{1}+1\right) \Gamma\left(\alpha_{1}-1\right)}\left|t_{1}-t_{2}\right|\right] } \\
& \cdot\left(\mathcal{C}_{\Phi}\left\|x_{n}\right\|\left\|^{q_{1}}+m_{1}\right\| x_{n} \|+M_{\Phi}\right) \\
= & {\left[\frac{(q-1) M_{1}^{q-2}}{\Gamma\left(\alpha_{1}+\beta_{1}+1\right)}\left|t_{1}^{\alpha_{1}+\beta_{1}}-t_{2}^{\alpha_{1}+\beta_{1}}\right|+\frac{(q-1) M_{1}^{q-2}}{\Gamma\left(\alpha_{1}+\beta_{1}\right)}\left|t_{1}-t_{2}\right|\right]\left(\mathcal{C}_{\Phi}\left\|x_{n}\right\|\left\|^{q_{1}}+m_{1}\right\| x_{n} \|+M_{\Phi}\right), }
\end{aligned}
$$

where $B(\cdot, \cdot)$ is the Beta function. In the same manner, we have

$$
\begin{aligned}
& \left|U_{2}\left(x_{n}, y_{n}\right)\left(t_{1}\right)-U_{2}\left(x_{n}, y_{n}\right)\left(t_{2}\right)\right| \\
\leq & {\left[\frac{(q-1) M_{1}^{q-2}}{\Gamma\left(\alpha_{2}+\beta_{2}+1\right)}\left|t_{1}^{\alpha_{2}+\beta_{2}}-t_{2}^{\alpha_{2}+\beta_{2}}\right|+\frac{(q-1) M_{1}^{q-2}}{\Gamma\left(\alpha_{2}+\beta_{2}\right)}\left|t_{1}-t_{2}\right|\right]\left(\mathcal{C}_{\Psi}\left\|y_{n}\right\|\left\|^{q_{1}}+m_{2}\right\| y_{n} \|+M_{\Psi}\right) . }
\end{aligned}
$$


By (26) and (27), we have

$$
\begin{aligned}
& \left|U\left(x_{n}, y_{n}\right)\left(t_{1}\right)-U\left(x_{n}, y_{n}\right)\left(t_{2}\right)\right| \\
& =\left|U_{1}\left(x_{n}, y_{n}\right)\left(t_{1}\right)-U_{1}\left(x_{n}, y_{n}\right)\left(t_{2}\right)\right|+\left|U_{2}\left(x_{n}, y_{n}\right)\left(t_{1}\right)-U_{2}\left(x_{n}, y_{n}\right)\left(t_{2}\right)\right| \\
& \leq\left[\frac{(q-1) M_{1}^{q-2}}{\Gamma\left(\alpha_{1}+\beta_{1}+1\right)}\left|t_{1}^{\alpha_{1}+\beta_{1}}-t_{2}^{\alpha_{1}+\beta_{1}}\right|+\frac{(q-1) M_{1}^{q-2}}{\Gamma\left(\alpha_{1}+\beta_{1}\right)}\left|t_{1}-t_{2}\right|\right]\left(\mathcal{C}_{\Phi}\left\|x_{n}\right\|^{q_{1}}+m_{1}\left\|x_{n}\right\|+M_{\Phi}\right) \\
& \quad+\left[\frac{(q-1) M_{1}^{q-2}}{\Gamma\left(\alpha_{2}+\beta_{2}+1\right)}\left|t_{1}^{\alpha_{2}+\beta_{2}}-t_{2}^{\alpha_{2}+\beta_{2}}\right|+\frac{(q-1) M_{1}^{q-2}}{\Gamma\left(\alpha_{2}+\beta_{2}\right)}\left|t_{1}-t_{2}\right|\right]\left(\mathcal{C}_{\Psi}\left\|y_{n}\right\|^{q_{1}}+m_{2}\left\|y_{n}\right\|+M_{\Psi}\right) .
\end{aligned}
$$

Equation (28) tends to zero as $t_{1} \rightarrow t_{2}$. Therefore, $U=\left(U_{1}, U_{2}\right)$ is equi-continuous on $\mathcal{D}$. Using the Arzelá - Ascoli theorem, $U(\mathcal{D})$ is compact. Hence, $U$ is $\varsigma$-Lipschitz with constant zero. This completes the proof.

Theorem 13. Under the hypotheses $\left(H_{1}\right),\left(H_{3}\right),\left(H_{4}\right)$ and $\left(H_{5}\right)$, and if

$$
(q-1) M_{1}^{q-2} A_{1}+(q-1) M_{1}^{q-2} A_{2}+\mathcal{C}_{v}^{*} \leq 1,
$$

then the coupled system (2) has at least one solution $(x, y) \in \mathcal{H}$.

Proof. In view of Theorems 10-12, $U$ and $V$ are continuous and $\varsigma$-Lipschitz with constant $\mathcal{C}_{v}^{*}$ and 0 . By the help of Definition 4 , we have $W$ is strict $\varsigma$-contraction.

Let $S=\{(x, y) \in \mathcal{H}: \lambda \in[0,1] \mid(x, y)=\lambda W(x, y)\}$. Now, to prove that $S$ is bounded, we obtain

$$
\begin{aligned}
\|(x, y)\| & =\|\lambda W(x, y)\| \leq\|\lambda U(x, y)\|+\|\lambda V(x, y)\| \\
& \leq(q-1) M_{1}^{q-2}\left(A_{1}\|(x, y)\|^{q_{1}}+A_{2}\|(x, y)\|+A_{3}\right)+\mathcal{C}_{v}^{*}\|(x, y)\|^{p_{1}}+M_{v} \\
& \leq\left((q-1) M_{1}^{q-2}\left(A_{1}+A_{2}\right)+\mathcal{C}_{v}^{*}\right)\|(x, y)\|+(q-1) M_{1}^{q-2} A_{3}+M_{v} .
\end{aligned}
$$

Hence, the set of solutions $\mathcal{S}$ is bounded. Using Theorem 6, the coupled system (2) has at least one solution. This completes the proof.

Theorem 14. Suppose the hypotheses $\left(H_{1}\right)$ to $\left(H_{5}\right)$ are satisfied. Then, the coupled system (2) has a unique solution provided

$$
\Omega=A_{4}+A_{5}+\mathcal{C}_{v}<1
$$

where

$$
\begin{gathered}
A_{4}=\max \left\{\frac{(q-1) M_{1}^{q-2} m_{1}}{\Gamma\left(\alpha_{1}+\beta_{1}\right)}, \frac{(q-1) M_{1}^{q-2} m_{2}}{\Gamma\left(\alpha_{2}+\beta_{2}\right)}\right\}, \\
A_{5}=\frac{(q-1) M_{1}^{q-2} m_{\Phi}}{\Gamma\left(\alpha_{1}+\beta_{1}\right)}+\frac{(q-1) M_{1}^{q-2} m_{\Psi}}{\Gamma\left(\alpha_{2}+\beta_{2}\right)},
\end{gathered}
$$

and $\mathcal{C}_{v}$ is defined by (15).

Proof. Let $(x, y)$ and $(\bar{x}, \bar{y}) \in \mathcal{H}$ be two solutions, then

$$
\|W(x, y)-W(\bar{x}, \bar{y})\|=\|U(x, y)-U(\bar{x}, \bar{y})\|+\|V(x, y)-V(\bar{x}-\bar{y})\| .
$$


Using the conditions $\left(H_{1}\right),\left(H_{2}\right)$ and Theorem 7 , we obtain

$$
\begin{aligned}
\|U(x, y)-U(\bar{x}, \bar{y})\|= & \left\|U_{1}(x, y)-U_{1}(\bar{x}, \bar{y})\right\|+\left\|U_{2}(x, y)-U_{2}(\bar{x}, \bar{y})\right\| \\
= & \int_{0}^{1} G_{\alpha_{1}}(t, \xi) \mid L_{q}\left(I_{0}^{\beta_{1}} \mathcal{F}_{1}(\xi) x(\xi)+\Phi(\xi, x(\xi), y(\xi))\right) \\
& -L_{q}\left(I_{0}^{\beta_{1}} \mathcal{F}_{1}(\xi) \bar{x}(\xi)+\Phi(\xi, \bar{x}(\xi), \bar{y}(\xi))\right) \mid d \xi \\
& +\int_{0}^{1} G_{\alpha_{2}}(t, \xi) \mid L_{q}\left(I_{0}^{\beta_{2}} \mathcal{F}_{2}(\xi) y(\xi)+\Psi(\xi, x(\xi), y(\xi))\right) \\
& -L_{q}\left(I_{0}^{\beta_{2}} \mathcal{F}_{2}(\xi) \bar{y}(\xi)+\Psi(\xi, \bar{x}(\xi), \bar{y}(\xi))\right) \mid d \xi \\
\leq & \frac{(q-1) M_{1}^{q-2}}{\Gamma\left(\alpha_{1}+\beta_{1}\right)}\left[m_{1}\|x-\bar{x}\|+m_{\Phi}\|(x-\bar{x}, y-\bar{y})\|\right] \\
& +\frac{(q-1) M_{1}^{q-2}}{\Gamma\left(\alpha_{2}+\beta_{2}\right)}\left[m_{2}\|y-\bar{y}\|+m_{\Psi}\|(x-\bar{x}, y-\bar{y})\|\right] \\
\leq & \left(\frac{(q-1) M_{1}^{q-2} m_{1}}{\Gamma\left(\alpha_{1}+\beta_{1}\right)}\|x-\bar{x}\|+\frac{(q-1) M_{1}^{q-2} m_{2}}{\Gamma\left(\alpha_{2}+\beta_{2}\right)}\|y-\bar{y}\|\right) \\
& +\left(\frac{(q-1) M_{1}^{q-2}}{\Gamma\left(\alpha_{1}+\beta_{1}\right)} m_{\Phi}+\frac{(q-1) M_{1}^{q-2}}{\Gamma\left(\alpha_{2}+\beta_{2}\right)} m_{\Psi}\right)\|(x-\bar{x}, y-\bar{y})\| \\
\leq & \left(A_{4}+A_{5}\right)\|(x-\bar{x}, y-\bar{y})\|,
\end{aligned}
$$

where $A_{4}$ and $A_{5}$ are defined by (32) and (33). Furthermore,

$$
\begin{aligned}
\|V(x, y)-V(\bar{x}, \bar{y})\| & =\left\|V_{1}(x)-V_{1}(\bar{x})\right\|+\left\|V_{2}(y)-V_{2}(\bar{y})\right\| \\
& \leq \frac{\eta_{1} b^{\gamma_{1}} \mathbf{K}_{1}}{\Gamma\left(\gamma_{1}+1\right)}\|x-\bar{x}\|+\frac{\eta_{2} b^{\gamma_{2}} \mathbf{K}_{2}}{\Gamma\left(\gamma_{2}+1\right)}\|y-\bar{y}\| \\
& \leq \mathcal{C}_{v}\|(x-\bar{x}, y-\bar{y})\| .
\end{aligned}
$$

Hence, from (34) and (35), we have

$$
\|W(x, y)-W(\bar{x}, \bar{y})\| \leq\left(A_{4}+A_{5}+\mathcal{C}_{v}\right)\|(x-\bar{x}, y-\bar{y})\|,
$$

which implies that the operator $W$ is contraction due to (31). By the Banach fixed point theorem, system (2) has a unique solution. This completes the proof.

\section{Hyers-Ulam Stability}

In this section, we study Hyers-Ulam stability for the coupled system of fractional differential equations with p-Laplacian operator (2). Using Definitions 6 and 7 and Theorems 6 and 8 , the corresponding results are received.

Theorem 15. Suppose that the assumptions $\left(H_{1}\right)$ to $\left(H_{5}\right)$ and (31) hold, and the matrix $Q$ is converging to zero, the solutions of the system are Hyers-Ulam stable.

Proof. Let $(x, y)$ and $\left(x^{*}, y^{*}\right) \in \mathcal{H}$ be two solutions and define operator $W=\left(W_{1}, W_{2}\right)$ and $W_{1}=U_{1}+V_{1}, W_{2}=U_{2}+V_{2}$. In view of Theorem 14, we have

$$
\begin{aligned}
\left\|W_{1}(x, y)-W_{1}\left(x^{*}, y^{*}\right)\right\| \leq & \left\|U_{1}(x, y)-U_{1}\left(x^{*}, y^{*}\right)\right\|+\left\|V_{1} x-V_{1} x^{*}\right\| \\
\leq & {\left[\frac{(q-1) M_{1}^{q-2}\left(m_{1}+m_{\Phi}\right)}{\Gamma\left(\alpha_{1}+\beta_{1}\right)}+\frac{\eta_{1} b^{\gamma_{1}} \mathbf{K}_{1}}{\Gamma\left(\gamma_{1}+1\right)}\right]\left\|x-x^{*}\right\| } \\
& +\frac{(q-1) M_{1}^{q-2} m_{\Phi}}{\Gamma\left(\alpha_{1}+\beta_{1}\right)}\left\|y-y^{*}\right\|=C_{1}\left\|x-x^{*}\right\|+C_{2}\left\|y-y^{*}\right\|,
\end{aligned}
$$


where

$$
C_{1}=\frac{(q-1) M_{1}^{q-2}\left(m_{1}+m_{\Phi}\right)}{\Gamma\left(\alpha_{1}+\beta_{1}\right)}+\frac{\eta_{1} b^{\gamma_{1}} \mathbf{K}_{1}}{\Gamma\left(\gamma_{1}+1\right)}
$$

and

$$
C_{2}=\frac{(q-1) M_{1}^{q-2} m_{\Phi}}{\Gamma\left(\alpha_{1}+\beta_{1}\right)} .
$$

Similarly, we can also obtain

$$
\begin{aligned}
\left\|W_{2}(x, y)-W_{2}\left(x^{*}, y^{*}\right)\right\| \leq & \frac{(q-1) M_{1}^{q-2} m_{\Psi}}{\Gamma\left(\alpha_{2}+\beta_{2}\right)}\left\|x-x^{*}\right\| \\
& +\left[\frac{(q-1) M_{1}^{q-2}\left(m_{2}+m_{\Psi}\right)}{\Gamma\left(\alpha_{2}+\beta_{2}\right)}+\frac{\eta_{2} b^{\gamma_{2}} \mathbf{K}_{2}}{\Gamma\left(\gamma_{2}+1\right)}\right]\left\|y-y^{*}\right\| \\
= & C_{3}\left\|x-x^{*}\right\|+C_{4}\left\|y-y^{*}\right\|,
\end{aligned}
$$

where

$$
C_{3}=\frac{(q-1) M_{1}^{q-2} m_{\Psi}}{\Gamma\left(\alpha_{2}+\beta_{2}\right)}
$$

and

$$
C_{4}=\frac{(q-1) M_{1}^{q-2}\left(m_{2}+m_{\Psi}\right)}{\Gamma\left(\alpha_{2}+\beta_{2}\right)}+\frac{\eta_{2} b^{\gamma_{2}} \mathbf{K}_{2}}{\Gamma\left(\gamma_{2}+1\right)} .
$$

So from (36) and (37), we obtain the inequalities given below

$$
\begin{aligned}
& \left\|W_{1}(x, y)-W_{1}\left(x^{*}, y^{*}\right)\right\| \leq C_{1}\left\|x-x^{*}\right\|+C_{2}\left\|y-y^{*}\right\|, \\
& \left\|W_{2}(x, y)-W_{2}\left(x^{*}, y^{*}\right)\right\| \leq C_{3}\left\|x-x^{*}\right\|+C_{4}\left\|y-y^{*}\right\| .
\end{aligned}
$$

From (38), we obtain the following inequality

$$
\left\|W(x, y)-W\left(x^{*}, y^{*}\right)\right\| \leq Q\left\|(x, y)-\left(x^{*}, y^{*}\right)\right\|,
$$

where $Q=\left(\begin{array}{ll}C_{1} & C_{2} \\ C_{3} & C_{4}\end{array}\right)$. Since $Q$ converges to zero, system (2) is Hyers-Ulam stable. This completes the proof.

\section{Examples}

Example 1. Consider the following coupled system of fractional differential equations with $p$ Laplacian operator and fractional order differential and integral initial and boundary conditions of the type

$$
\left\{\begin{array}{l}
D_{0}^{\frac{1}{2}} L_{5}\left(D_{0}^{\frac{5}{2}} x(t)\right)-\frac{e^{-2 t} x(t)}{20}=\frac{t^{2}+\sin |x(t)|+\cos |y(t)|}{100}, \\
D_{0}^{\frac{1}{2}} L_{5}\left(D_{0}^{\frac{5}{2}} y(t)\right)-\frac{t y(t)}{30}=\frac{\cos |x(t)|+\sin |y(t)|}{50+t^{2}}, \\
\left.D_{0}^{\frac{5}{2}} x(t)\right|_{t=0}=0, x(0)=0, x^{\prime \prime}(0)=0, \\
x^{\prime}(t)=\frac{1}{3 \Gamma\left(\frac{3}{2}\right)} \int_{0}^{1}(1-\xi)^{\frac{3}{2}-1} \cos (x(\xi)) d \xi, \\
\left.D_{0}^{\frac{5}{2}} y(t)\right|_{t=0}=0, y(0)=0, y^{\prime \prime}(0)=0, \\
y^{\prime}(t)=\frac{1}{3 \Gamma\left(\frac{3}{2}\right)} \int_{0}^{1}(1-\xi)^{\frac{1}{2}-1} \cos (y(\xi)) d \xi,
\end{array}\right.
$$


where $p=5, q=\frac{5}{4}, t \in[0,1], \alpha_{1}=\alpha_{2}=\frac{5}{2}, \beta_{1}=\beta_{2}=\frac{1}{2}, \eta_{1}=\eta_{2}=\frac{1}{3}, \gamma_{1}=\frac{3}{2}, \gamma_{2}=\frac{1}{2}$, $b=1$. From this system, we have

$$
\begin{aligned}
& \mathcal{F}_{1}(t)=\frac{e^{-2 t}}{20}, \mathcal{F}_{2}(t)=\frac{t}{30}, \\
& \Phi(t, x(t), y(t))=\frac{t^{2}+\sin |x(t)|+\cos |y(t)|}{100}, \text { and } \\
& \Psi(t, x(t), y(t))=\frac{\cos |x(t)|+\sin |y(t)|}{50+t^{2}} .
\end{aligned}
$$

For any $(x, y),(\bar{x}, \bar{y}) \in \mathcal{H}, t \in[0,1]$, we have

$$
\begin{aligned}
& m_{\Phi}=\frac{3}{100}, m_{\Psi}=\frac{1}{50}, \\
& |\Phi(t, x, y)-\Phi(t, \bar{x}, \bar{y})| \leq \frac{3}{100}\|(x-\bar{x}, y-\bar{y})\|, \\
& |\Psi(t, x, y)-\Psi(t, \bar{x}, \bar{y})| \leq \frac{1}{50}\|(x-\bar{x}, y-\bar{y})\|,
\end{aligned}
$$

and $\mathcal{C}_{\Phi}=\frac{1}{10}, \mathcal{C}_{\Psi}=\frac{1}{10}, M_{\Phi}=\frac{1}{100}, M_{\Psi}=\frac{1}{50}, q_{1}=\frac{1}{2}$,

$$
\begin{aligned}
& \|\Phi(t, x, y)\| \leq \frac{1}{10}\|x\|^{\frac{1}{2}}+\frac{1}{100} \\
& \|\Psi(t, x, y)\| \leq \frac{1}{10}\|y\|^{\frac{1}{2}}+\frac{1}{50} .
\end{aligned}
$$

Furthermore, we can obtain $m_{1}=\frac{1}{20}, m_{2}=\frac{1}{30}, M_{1}=2, \mathbf{K}_{1}=\frac{1}{100}, \mathbf{K}_{2}=\frac{1}{200}$. Then, we see that

$$
\Omega=A_{4}+A_{5}+\mathcal{C}_{v}=\frac{1}{80 \times 2^{\frac{3}{4}}}+\frac{1}{80 \times 2^{\frac{3}{4}}}+\frac{1}{300 \sqrt{\pi}}<1 .
$$

Using Theorem 14, the coupled system has a unique solution. By direct calculation, we obtain

$$
Q=\left(\begin{array}{cc}
\frac{1}{50 \times 2^{\frac{3}{4}}}+\frac{1}{300 \sqrt{\pi}} & \frac{3}{400 \times 2^{\frac{3}{4}}} \\
\frac{1}{200 \times 2^{\frac{3}{4}}} & \frac{1}{75 \times 2^{\frac{3}{4}}}+\frac{1}{600 \sqrt{\pi}}
\end{array}\right) \approx\left(\begin{array}{cc}
0.0138 & 0.0045 \\
0.0030 & 0.0088
\end{array}\right) .
$$

On calculation, we obtain the spectral radius

$$
\rho(Q)=\max \{|0.01031|,|-0.00013|\}=0.01031<1,
$$

which shows that the matrix $Q$ converges to zero, and using Theorem 15, the solutions of the problem (39) are Hyers-Ulam stable.

Example 2. Consider the following coupled system of fractional differential equations with $p$ Laplacian operator and fractional order differential initial and boundary conditions of the type 


$$
\left\{\begin{array}{l}
D_{0}^{\frac{1}{3}} L_{3}\left(D_{0}^{\frac{7}{3}} x(t)\right)-\frac{e^{-3 t} x(t)}{30}=\frac{\sin |x(t)|+\cos |y(t)|}{100+t^{3}} \\
D_{0}^{\frac{1}{3}} L_{3}\left(D_{0}^{\frac{7}{3}} y(t)\right)-\frac{t y(t)}{20}=\frac{\cos |x(t)|+\sin |y(t)|}{1000} \\
\left.D_{0}^{\frac{7}{3}} x(t)\right|_{t=0}=0, x(0)=0, x^{\prime \prime}(0)=0, \\
x^{\prime}(1)=\frac{1}{4 \Gamma\left(\frac{3}{2}\right)} \int_{0}^{1}(1-\xi)^{\frac{3}{2}-1} \sin (x(\xi)) d \xi \\
\left.D_{0}^{\frac{7}{3}} y(t)\right|_{t=0}=0, y(0)=0, y^{\prime \prime}(0)=0 \\
y^{\prime}(1)=\frac{1}{4 \Gamma\left(\frac{1}{2}\right)} \int_{0}^{1}(1-\xi)^{\frac{1}{2}-1} \sin (y(\xi)) d \xi
\end{array}\right.
$$

where $p=3, q=\frac{3}{2}, t \in[0,1], \alpha_{1}=\alpha_{2}=\frac{7}{3}, \beta_{1}=\beta_{2}=\frac{1}{3}, \eta_{1}=\eta_{2}=\frac{1}{4}, \gamma_{1}=\frac{3}{2}, \gamma_{2}=\frac{1}{2}$, $b=1$. So, we have

$$
\begin{aligned}
& \mathcal{F}_{1}(t)=\frac{e^{-3 t}}{30}, \mathcal{F}_{2}(t)=\frac{t}{20}, \\
& \Phi(t, x, y)=\frac{\sin |x(t)|+\cos |y(t)|}{100+t^{3}}, \text { and } \\
& \Psi(t, x, y)=\frac{\cos |x(t)|+\sin |y(t)|}{1000} .
\end{aligned}
$$

For any $(x, y),(\bar{x}, \bar{y}) \in \mathcal{H}, t \in[0,1]$, we have

$$
\begin{aligned}
& m_{\Phi}=\frac{1}{100}, m_{\Psi}=\frac{1}{50}, \\
& |\Phi(t, x, y)-\Phi(t, \bar{x}, \bar{y})| \leq \frac{1}{100}\|(x-\bar{x}, y-\bar{y})\|, \\
& |\Psi(t, x, y)-\Psi(t, \bar{x}, \bar{y})| \leq \frac{1}{50}\|(x-\bar{x}, y-\bar{y})\|,
\end{aligned}
$$

and $\mathcal{C}_{\Phi}=\frac{1}{100}, \mathcal{C}_{\Psi}=\frac{1}{100}, M_{\Phi}=\frac{1}{50}, M_{\Psi}=\frac{1}{500}, q_{1}=\frac{1}{2}$,

$$
\begin{aligned}
& \|\Phi(t, x, y)\| \leq \frac{1}{100}\|x\|^{\frac{1}{2}}+\frac{1}{50} \\
& \|\Psi(t, x, y)\| \leq \frac{1}{100}\|y\|^{\frac{1}{2}}+\frac{1}{500} .
\end{aligned}
$$

Furthermore, we can also obtain $m_{1}=\frac{1}{30}, m_{2}=\frac{1}{20}, M_{1}=2, \mathbf{K}_{1}=\frac{1}{100}, \mathbf{K}_{2}=\frac{1}{200}$. Then, we see that

$$
\Omega=A_{4}+A_{5}+\mathcal{C}_{v}=\frac{1}{40 \times 2^{\frac{1}{2}} \Gamma\left(\frac{2}{3}\right)}+\frac{1}{200 \times 2^{\frac{1}{2}} \Gamma\left(\frac{2}{3}\right)}+\frac{1}{400 \sqrt{\pi}}<1 .
$$

Using Theorem 14, the coupled system has a unique solution. Upon calculation, we obtain

$$
Q=\left(\begin{array}{cc}
\frac{13}{600 \sqrt{2} \Gamma\left(\frac{2}{3}\right)}+\frac{1}{400 \Gamma\left(\frac{1}{2}\right)} & \frac{1}{200 \sqrt{2} \Gamma\left(\frac{2}{3}\right)} \\
\frac{1}{100 \sqrt{2} \Gamma\left(\frac{2}{3}\right)} & \frac{7}{200 \sqrt{2} \Gamma\left(\frac{2}{3}\right)}+\frac{1}{800 \sqrt{\pi}}
\end{array}\right) \approx\left(\begin{array}{cc}
0.0184 & 0.0039 \\
0.0078 & 0.0281
\end{array}\right) .
$$

Direct calculation implies the spectral radius

$$
\rho(Q)=\max \{|0.0306|,|0.0159|\}=0.0306<1,
$$

which shows that the matrix $Q$ converges to zero, and using Theorem 15, the solutions of the problem (40) are Hyers-Ulam stable. 


\section{Conclusions}

In this paper, we use the coincidence degree method and nonlinear functional analysis theory to deal with the existence and uniqueness of solutions and the matrix eigenvalue method in order to investigate Hyers-Ulam stability for a coupled system of fractional differential equations with nonlinear $p$-Laplacian operator. Since the system we studied is more extensive and the initial boundary value conditions used are different from those used in references $[6,14,15]$, the proofs may be carried out in the case of q-difference similarly.

Author Contributions: Conceptualization, J.S. and B.G.; data curation, B.G.; formal analysis, B.G.; funding acquisition, J.S. and B.G.; investigation, B.G.; methodology, J.S.; project administration, B.G.; validation, J.S. and B.G.; writing original draft, J.S. and B.G.; writing review and editing, J.S. and B.G. All authors read and agreed to the published version of the manuscript.

Funding: Project supported by the NNSF of China (Grant Nos 11731014 and 11571254), NSF of Shandong (Grant No ZR2018LA004).

Institutional Review Board Statement: Not applicable.

Informed Consent Statement: Not applicable.

Data Availability Statement: Not applicable.

Acknowledgments: The authors sincerely thank the referees for a number of constructive suggestions and corrections which have significantly improved the contents and the exposition of the paper.

Conflicts of Interest: The authors declare no conflict of interest.

\section{References}

1. Kilbas, A.A.; Srivastava, H.M.; Trujillo, J.J. Theory and Applications of Fractional Differential Equations, North-Holland Mathematics Studies; Elsevier Science B.V.: Amsterdam, The Netherlands, 2006; Volume 204.

2. Urs, C. Ulam-Hyers stability for coupled fixed points of contractive type operators. J. Nonlinear Sci. Appl. 2013, 6, 124-136.

3. Podlubny, I. Fractional differential equations. In Mathematics in Science and Engineering; Academic Press: San Diego, CA, USA, 1999; Volume 198

4. Prasad, K.P.; Krushna, B.M.B. Multiple positive solutions for a coupled system of $p$-Laplacian fractional order two-point boundary value problems. Int. J. Differ. Equ. 2014, 2014, 485647. [CrossRef]

5. Miller, K.S.; Ross, B. An Introduction to the Fractional Calculus and Fractional Differential Equations; Wiley: New York, NY, USA, 1993.

6. Khan, A.; Li, Y.; Shah, K.; Khan, T.S. On coupled $p$-Laplacian fractional differential equations with nonlinear boundary conditions, Complexity 2017, 2017, 8197610. [CrossRef]

7. Li, F.; Bao, Y. Uniform stability of the solution for a Memory-type elasticity system with nonhomogeneous boundary control condition. J. Dyn. Control Syst. 2017, 23, 301-315. [CrossRef]

8. Wang, J.; Shah, K.; Ali, A. Existence and Hyers-Ulam stability of fractional nonlinear impulsive switched coupled evolution equations. Math. Meth. Appl. Sci. 2018, 41, 2392-2402. [CrossRef]

9. Gao, L.; Wang, D.; Wang, G. Further results on exponential stability for impulsive switched nonlinear time delay systems with delayed impulse effects. Appl. Math. Comput. 2015, 268, 186-200. [CrossRef]

10. Leibenson, L.S. General problem of the movement of a compressible fluid in a porous medium. Izv. Akad. Nauk Kirg. SSR 1983, 9, 7-10. (In Russian)

11. Khamessi, B.; Hamiaz, A. Existence and exact asymptotic behaviour of positive solutions for fractional boundary value problem with $p$-Laplacian operator. J. Taibah Univ. Sci. 2019, 13, 370-376. [CrossRef]

12. Bai, C. Existence and uniqueness of solutions for fractional boundary value problems with $p$-Laplacian operator. Adv. Differ. Equ. 2018, 2018, 4. [CrossRef]

13. Lu, H.; Han, Z.; Sun, S. Multiplicity of positive solutions for Sturm-Liouville boundary value problems of fractional differential equations with $p$-Laplacian. Bound. Value Probl. 2014, 2014, 26. [CrossRef]

14. Khan, H.; Li, Y.; Sun, H.; Khan, A. Existence of solution and Hyers-Ulam stability for a coupled system of fractional differential equations with $p$-Laplacian operator. J. Nonlinear Sci. Appl. 2017, 10, 5219-5229. [CrossRef]

15. Khan, H.; Li, Y.; Chen, W.; Baleanu, D.; Khan, A. Existence theorems and Hyers-Ulam stability for a coupled system of fractional differential equations with $p$-Laplacian operator. Bound Value Probl. 2017, 2017, 157. [CrossRef]

16. Urs, C. Coupled fixed point theorem and applications to periodic boundary value problem. Miskolic Math Notes 2013, 14, 323-333. [CrossRef]

17. Hilfer, R. Applications of Fractional Calculus in Physics; World Scientific: Singapore, 2000. 
18. Bai, Z.; Lv, H. Positive solutions for boundary value problem of nonlinear fractional differential equation. J. Math. Anal. Appl. 2005, 311, 495-505. [CrossRef]

19. Isaia, F. On a nonlinear integral equation without compactness. Acta Math. Univ. Comenian. 2006, 75, $233-240$.

20. Shen, T.; Liu, W.; Shen, X. Existence and uniqueness of solutions for several BVPs of fractional differential equations with p-Laplacian operator. Mediterr. J. Math. 2016, 13, 4623-4637. [CrossRef] 